\title{
Seguridad y migración en las fronteras de México: diagnóstico y recomendaciones de política y cooperación regional
}

\author{
GUADALUPE CORREA-CABRERA*
}

RESUMEN: El presente documento presenta un diagnóstico de las dos fronteras de México - la frontera México-Estados Unidos y la frontera sur (México-Centroamérica) - en relación con el tráfico de drogas, la violencia vinculada al crimen organizado, la migración indocumentada y el tráfico de migrantes. También hace un recuento y breve evaluación de las políticas que se han implementado recientemente para hacer frente a dichos problemas, así como de los resultados de estas implementaciones. Este análisis comparativo sirve de base para elaborar recomendaciones de política que Estados Unidos, México y los gobiernos de Centroamérica deberían impulsar para fortalecer la seguridad humana y la cooperación regional en estos temas. Se concluye que los principales problemas en materia de seguridad fronteriza en estas dos regiones del hemisferio solamente podrán abordarse a través de la cooperación de los países involucrados y el diseño de verdaderas políticas de desarrollo humano.

PALABRAS ClAVE: seguridad, migración, crimen organizado, frontera MéxicoEstados Unidos, frontera sur.

* Profesora-investigadora y directora del Departamento de Gobierno de la Universidad de Texas, Estados Unidos. 
ABSTRACT: This article presents an analysis of Mexico's two borders - the Mexico-US border and the southern border (Mexico-Central America)with regards to drug trafficking, violence related to organized crime, undocumented migration, and migrant smuggling. It also provides a brief account and assessment of the policies that have been recently implemented with the aim of solving these problems. This comparative analysis is used as a starting point to elaborate recommendations of policies that the United States, Mexico, and Central American governments should implement in order to strengthen human security as well as regional cooperation in these areas. This article concludes by asserting that the main problems regarding border security in these two regions of the hemisphere would only be solved through the close cooperation between the nations involved and the design of effective human development policies.

KEY WORDS: security, migration, organized crime, Mexico-U.S. border, southern border. 


\section{INTRODUCCIÓN}

ctualmente, las dos fronteras de México son espacios de gran im-
portancia económica y estratégica para gran parte de los países
del hemisferio donde el tema de la seguridad adquiere un papel
esencial. La frontera norte limita con los estados de California, Arizona, Nuevo México y Texas, ${ }^{1}$ mientras que la frontera sur — denominada aquí frontera México-Centroamérica-incluye los estados de Quintana Roo, Campeche, Tabasco y Chiapas, que limitan con Belice y Guatemala. ${ }^{2}$ Estas dos fronteras mantienen diferencias importantes en materia de demografía, espacio geográfico, desarrollo económico y producción. Sin embargo, ambas regiones enfrentan serios problemas comunes de seguridad que requieren una atención inmediata y acciones de cooperación regional.

En particular, las dos fronteras de México, a pesar de sus marcadas diferencias, enfrentan problemáticas entre las que destacan el narcotráfico, la violencia del crimen organizado y el tráfico de migrantes y de armas. Estas actividades del crimen organizado se concentran en las zonas fronterizas y operan de la siguiente manera:

Desde Centroamérica y América del Sur ingresan a México drogas, armas pequeñas y delincuentes. México, a su vez, exporta sus cárteles a Centroamérica, principalmente los de Sinaloa, el del Golfo y los Zetas, que actúan en Guatemala. Al mismo tiempo, la población transmigrante sufre la extorsión de los traficantes y de las autoridades gubernamentales corruptas. Asimismo, México envía grandes cantidades de narcóticos hacia Estados Unidos, de donde proviene la demanda de drogas y el financiamiento para que ese comercio ilegal sea posible, además de otro factor que alimenta a toda la criminalidad común y organizada de México: las armas, debido a las facilidades para la venta libre en territorio estadounidense (Benítez, 2011: 184).

${ }^{1}$ La frontera México-Estados Unidos tiene una extensión de aproximadamente 3,152 kilómetros.

2 Dicha frontera posee una extensión de 1,149 kilómetros, de los cuales 956 limitan con Guatemala y 193 con Belice. En el presente trabajo se utiliza el término frontera MéxicoCentroamérica dada la importancia de esta zona no únicamente para Guatemala y Belice sino para el resto de la región centroamericana. 
En épocas recientes, el vínculo entre el crimen organizado y la migración indocumentada adquiere especial relevancia y se agudiza en los primeros años del presente siglo al aparecer rutas de narcotráfico que coinciden con las de los migrantes en su trayecto hacia Estados Unidos. Los migrantes enfrentan riesgos muy graves — especialmente los grupos más vulnerables (mujeres y menores de edad) - entre los que destacan la extorsión, secuestro, violación y asesinato (Armijo, 2011a). Así, «el tráfico de migrantes, principalmente centro y sudamericanos, se ha vuelto un grave asunto humanitario». En este contexto, "los migrantes son víctimas de las organizaciones criminales ya que se ven orillados a utilizar sus servicios, debido a las dificultades derivadas del mayor control de la frontera sur de México, de las rutas de migración y de la frontera sur de Estados Unidos» (Benítez, 2011: 179). El fenómeno resultante puede describirse de la siguiente forma:

El crimen organizado va cooptando - y en ocasiones raptando - migrantes en dichas rutas de forma creciente, convirtiéndose en un problema de seguridad pública y de derechos humanos para el Estado mexicano. Al respecto, cabe señalar que la Comisión Nacional de Derechos Humanos así como múltiples organizaciones no gubernamentales defensoras de los derechos de los migrantes han denunciado importantes violaciones a los derechos de los extranjeros durante su paso por México. De igual manera, los gobiernos de América Central han denunciado la desaparición de gran cantidad de personas víctimas de las organizaciones criminales, como los Zetas y el Cártel del Golfo, pues en las rutas carreteras que parten de Chiapas hacia Texas por los estados de Oaxaca, Tabasco, Veracruz y Tamaulipas se ha detectado esta actividad de forma intensa. De esta manera, se ha securitizado el tema de la migración tanto en Estados Unidos como en México y Centroamérica (Benítez, 2011: 181).

Durante los últimos años, las políticas de migración y de seguridad parecen estar cada vez más estrechamente relacionadas. A partir de los ataques del 11 de septiembre de 2001 en Estados Unidos, «la migración ha ocupado un lugar cada vez más relevante en las políticas de seguridad, bajo el argumento de prevenir la entrada de posibles terroristas». Este proceso, que se denomina securitización de la migración es particularmente visible en Estados

$150 \frac{\text { PRIMER SEMESTRE } 2014}{\text { MIGRACIÓN Y DESARROLLO NÚM. } 22}$ 
Unidos, y se ha extendido a las políticas migratorias de México y Centroamérica (Armijo, 2011a: 5).

No obstante, la presencia de actores y problemáticas comunes en el tema de la seguridad fronteriza, existen enormes diferencias en la protección de las fronteras que México comparte con Estados Unidos y Guatemala/ Belice. El papel de Estados Unidos es crucial, pues define algunas pautas que afectan a todos los países de la región. Gran parte de las políticas de seguridad fronteriza en la región que divide a México y Estados Unidos se diseñan de manera unilateral, y es posible afirmar que la cooperación con México y Centroamérica en esta materia se define en su mayor parte en Estados Unidos. Asimismo, las políticas de seguridad fronteriza en la frontera MéxicoEstados Unidos — centradas en la seguridad del Estado, como consecuencia del incremento de las presiones para reforzar la seguridad después del 11 de septiembre de 2001 - han tenido un impacto muchas veces negativo en la frontera México-Centroamérica.

Las políticas de seguridad en las dos fronteras de México presentan grandes limitaciones y muestran la necesidad de una mayor colaboración entre las naciones involucradas en la problemática de la región. Es, por lo tanto, necesario repensar el diseño de soluciones a los grandes problemas que afectan de manera significativa a la sociedad en esta parte del hemisferio, como son el tráfico de drogas, de armas y de personas, la violación de derechos humanos y la violencia extrema en la era de la denominada "guerra contra las drogas».

El presente documento presenta un diagnóstico de las dos fronteras de México - la frontera México-Estados Unidos y la frontera sur (MéxicoCentroamérica) - respecto al tráfico de drogas, la violencia, la migración indocumentada y el tráfico de migrantes, así como un recuento y breve evaluación de las políticas de seguridad que se han implementado recientemente para hacer frente a dichos problemas. Este análisis comparativo sirve de base para elaborar recomendaciones de política que tanto México como los gobiernos de Centroamérica deben impulsar para fortalecer la cooperación regional en estos temas. Con esta idea en mente, la primera parte del presente trabajo muestra un diagnóstico de la problemática de seguridad en 
la frontera México-Estados Unidos, al tiempo que describe y evalúa las políticas implementadas en fechas recientes para atender la situación en esta parte del continente. El mismo análisis se hace a continuación para la frontera México-Centroamérica. Finalmente, se mencionan algunas recomendaciones que tanto México como los gobiernos de Centroamérica podrían impulsar para fortalecer la cooperación regional en materia de seguridad fronteriza.

\section{TRÁFICO DE DROGAS, VIOLENCIA Y MIGRACIÓN INDOCUMENTADA EN LA fRontera México-Estados Unidos}

Diagnóstico de la frontera norte de México

En las últimas décadas, los estados fronterizos del norte de México se han visto afectados por varios fenómenos, entre los que destacan la apertura comercial en un nuevo esquema de globalización y, de manera particular, el aumento exponencial de la violencia relacionada con el crimen organizado. En este contexto, también se observan rezagos en la provisión de servicios públicos en diversas ciudades fronterizas, principalmente en aquellas donde el crecimiento ha sido rápido dado el desarrollo de la industria maquiladora. Al mismo tiempo, la apertura comercial ha tenido cierto impacto en el aumento del tráfico de drogas hacia Estados Unidos. Pero, sobre todo, es importante destacar los efectos negativos de los niveles extremos de violencia ocasionados por el crimen organizado sobre la seguridad y la economía de los estados fronterizos mexicanos. Entre los factores que han contribuido a elevar los niveles de violencia en el lado mexicano de la frontera norte se incluyen el tráfico de drogas, la estrategia del gobierno federal en su lucha contra el crimen organizado, el tráfico de migrantes y sus nuevos vínculos con las organizaciones criminales y el tráfico de armas.

En los últimos años, y a pesar de las medidas tomadas para hacer frente a esta problemática, no se ha observado una baja considerable del tráfico de drogas por la frontera norte de México, al tiempo que se advierte un incre-

$152 \frac{\text { PRIMER SEMESTRE } 2014}{\text { MIGRACIÓN Y DESARROLLO NÚM. } 22}$ 
mento muy significativo en el tráfico de armas hacia el sur del continente, y hasta muy recientemente, una ligera disminución de los flujos migratorios, y sobre todo de la migración irregular o indocumentada.

La evidencia existente muestra que la extrema violencia que se ha registrado en México en años recientes ha beneficiado a las ciudades fronterizas estadounidenses, mientras que ha tenido un claro impacto negativo en la parte mexicana. Los efectos positivos de la reciente ola de violencia en México sobre la seguridad y economía fronterizas en el lado estadounidense son evidentes, no obstante el discurso público estadounidense y la información que presentan algunos medios de este país alegando lo contrario (Correa, 2013).

Las políticas

Después del 11 de septiembre de 2001 se produjo en Estados Unidos una reforma total del sistema de seguridad nacional para combatir la amenaza terrorista. ${ }^{3}$ En general, a partir de este momento, se observa una fuerte intensificación de la vigilancia en la zona fronteriza, así como el endurecimiento de los controles a la migración indocumentada. Según explica Benítez (2011), dos son los ejes de este cambio en la política de seguridad nacional en Estados Unidos: la Defensa de la Patria (Homeland Security) y la Doctrina de Acción Preventiva (Preemptive Action):

ambas están íntimamente vinculadas; sin embargo, la primera tiene como prioridad la defensa del territorio y la población de Estados Unidos e implica una reordenación burocrática total, mientras que la segunda se dirige a las acciones

${ }^{3}$ En este nuevo contexto se aprueban un serie de leyes entre las que destacan: la Ley de Seguridad Interna (Homeland Security Act, 2002), la Ley Patriota (USA PATRIOT Act, 2001), la Ley de Seguridad Fronteriza Reforzada y la Reforma de Visas de Entrada (Enhanced Border Security and Visa Entry Reform Act o EBSVERA, 2002), la Ley de Reforma en materia de Inteligencia y Prevensión del Terrorismo (Intelligence Reform and Terrorism Prevention Act, 2004), la Ley de Identificación Verdadera (Real ID Act, 2005) y la Ley del Cerco Seguro (Secure Fence Act, 2006). 
de política exterior: diplomáticas, militares, de cooperación económica, de inteligencia, etcétera. En este contexto, la frontera con México se convierte en un tema de gran relevancia para los intereses de seguridad de Estados Unidos (186).

En 2002, México firma con su vecino del norte el acuerdo «Fronteras inteligentes» bajo el cual se formaliza la cooperación antiterrorismo entre las dos naciones y se consolida la participación de todo el sistema de seguridad nacional mexicano - incluyendo las agencias encargadas de la seguridad pública, procuración de justicia, inteligencia y política migratoria - en apoyo a la estrategia de seguridad de Estados Unidos. Como parte de este nuevo esquema, se ayudó a proteger la frontera principalmente en sus puntos críticos (Tijuana-San Diego, Ciudad Juárez-El Paso y Nuevo Laredo-Laredo) respondiendo a los requerimientos estadounidenses de control de tránsito de personas y bienes. Asimismo, y de manera extraoficial, «se fortalecieron al máximo los intercambios de información sobre tránsito de extranjeros a nivel migratorio y de inteligencia, y se cooperó intensamente con las agencias de imposición de la ley de Estados Unidos (FBI, DEA, etcétera)» (Benítez, 2011: 186).

La nueva lógica de seguridad fronteriza se basa en la prevención del delito y de la migración ilegal - lo que se conoce en inglés como enforcement through deterrence. En este nuevo esquema, cabe destacar la asignación de cantidades importantes de recursos para prevenir la migración irregular o indocumentada, así como el tráfico de bienes ilícitos. Con este objetivo, se incrementan los presupuestos, la tecnología empleada y el número de funcionarios federales para proteger la frontera. Como parte de dicho esquema, destacan los incrementos significativos en el personal de la Patrulla Fronteriza (de menos de 3,000, en 1981, a 9,100, en 2001, para llegar a los más de 20,000 en 2010); la construcción de 650 millas de muro fronterizo desde 2006; un mayor uso de la tecnología en zonas de mayor tráfico; así como un aumento considerable en el número de deportaciones (Rosemblum y Brick, 2011). Es importante resaltar el hecho de que en Estados Unidos la migración se percibe como un tema de seguridad y de política interna.

$154 \frac{\text { PRIMER SEMESTRE } 2014}{\text { MIGRACIÓN Y DESARROLLO NÚM. } 22}$ 
En la era de la denominada "guerra contra las drogas» y derivado del aumento exponencial de la violencia en México relacionada con el crimen organizado, se consolida la posición estadounidense de reforzar la seguridad en su frontera con México y, bajo esta lógica, se endurecen las medidas para contrarrestar la migración «ilegal». En algunos estados de Estados Unidos se discute la aplicación de leyes restrictivas de los derechos de los inmigrantes indocumentados y en algunos casos se criminaliza esta situación. Tal es el ejemplo de la ley SB-1070 en Arizona, que resulta de una estrategia que en inglés se conoce como attrition through enforcement, es decir, la aplicación estricta de la ley con el objetivo de desincentivar la migración indocumentada. Legislaciones de este tipo conducen a destacar constantemente la condición de «ilegalidad» de la población inmigrante y al establecimiento de condiciones para lograr la deportación de este grupo.

Con la reciente aparición y multiplicación de estas posiciones en contra de la migración indocumentada, se desata una especie de conflicto entre los gobiernos de Estados Unidos, México y Centroamérica sobre la política migratoria y su reforma. El debate es especialmente complicado al interior de Estados Unidos. Por consiguiente, las posibilidades de implementar una legislación federal comprehensiva a favor de la regularización de los indocumentados en este país norteamericano parecen alejarse. Benítez (2011) describe esta situación de la siguiente manera:

Aunque la administración del presidente Barack Obama, iniciada en enero de 2009, ha tratado de impulsar en el nivel federal la idea de una legislación incluyente que procure la regularización de los inmigrantes indocumentados, ello no ha podido concretarse ... [Esta situación] conduce no sólo a favorecer las tendencias antimigración sino a vincular el problema con la seguridad nacional, donde incluso hay voces influyentes que sostienen que se debe emplear una fuerza directa de intervención en México con Marines para combatir a las organizaciones criminales en las ciudades mexicanas de la frontera, aduciendo la incapacidad de las autoridades de México (188).

En el contexto de la denominada "guerra contra las drogas» y de violencia extrema en México, las políticas de seguridad fronteriza han sido 
mayormente unilaterales y la dirección de la agenda en esta materia parece haber sido fijada, en gran parte, desde Washington. Sin embargo, se observa una cierta colaboración en el marco de la denominada Iniciativa Mérida, la cual plantea como objetivo «superar las limitaciones de la cooperación binacional antidrogas de años pasados» bajo un nuevo esquema «que reconoce las responsabilidades compartidas de Estados Unidos y México en la lucha contra la violencia que amenaza a los ciudadanos de ambos países generada por el narcotráfico». Así, «la Iniciativa Mérida proporciona habilidades técnicas y asistencia en territorio mexicano para la profesionalización de la policía, la reforma judicial y penitenciaria, el reforzamiento de las tecnologías de información, el desarrollo de infraestructura, la seguridad fronteriza y el fomento de una cultura de legalidad» (Ramos, 2011: 82-83). Cuatro son los objetivos estratégicos de la Iniciativa Mérida:

El primero es actuar para acotar el poder de los cárteles y la impunidad y protección de que gozan por parte de autoridades corruptas. Para ello se necesitaba como primer paso fortalecer todos los organismos que integran el sistema de seguridad del Estado mexicano, conformado principalmente por las fuerzas armadas, la Policía Federal, los organismos de inteligencia y todo el sistema de justicia penal. Como segundo propósito, la Iniciativa Mérida pretende fortalecer el control de la frontera México-Estados Unidos mediante el uso intensivo de tecnología. El tercero es mejorar la capacidad de los sistemas de justicia penal a través de reformas institucionales y administrativas para implementar los juicios orales. Y como cuarto aspecto, controlar las actividades de las pandillas asociadas al tráfico de drogas y disminuir su demanda. También la Iniciativa Mérida incluye el fortalecimiento de la sociedad civil a través de la Agencia para el Desarrollo Internacional (USAID, por sus siglas en inglés). El componente geopolítico se incluye con la expansión del proyecto a los países de Centroamérica y el Caribe y con propuestas para controlar también la frontera sur de México, considerada prácticamente abierta y con condiciones muy favorables para el desarrollo de las actividades criminales (Benítez, 2011: 189).

Según Benítez (2011), el propósito de la Iniciativa Mérida era

$156 \frac{\text { PRIMER SEMESTRE } 2014}{\text { MIGRACIÓN Y DESARROLLO NÚM. } 22}$ 
lograr fortalecer las capacidades de las instituciones del gobierno federal para el combate al narcotráfico, la lucha contra la corrupción, el acceso a nuevas tecnologías de detección y el equipamiento a las fuerzas armadas y la Policía Federal. La cooperación se [centraba] en la transferencia de equipo de alta tecnología, principalmente militar e informático, al igual que en el intercambio de información y en programas de capacitación para militares, policías y jueces (189). ${ }^{4}$

\section{Evaluación de las políticas}

Las políticas de seguridad en la frontera México-Estados Unidos, así como la cooperación bilateral para resolver la problemática fronteriza en los últimos años han tenido efectos limitados en general, y muy en particular en lo que se refiere al tráfico y consumo de drogas. Los avances en materia de combate a las drogas han sido extremadamente lentos. La creación del Departamento de Seguridad Interna (Department of Homeland Security) y la implementación de otras políticas «post-11 de septiembre» parecen no haber tenido un efecto visible sobre la eficacia de la política antidroga fronteriza con México. Es posible afirmar que en los últimos años las políticas antinarcóticos estadounidenses, si bien han asegurado coyunturalmente la cooperación de México, no han logrado reducir sustancialmente la oferta de drogas ni el papel de los grupos delictivos, por lo que el mercado ha resultado fortalecido y los grupos criminales han proliferado.

Lo anterior se debe, quizás, a que no se ha planteado una acción más integral vinculada tanto a la política de intercepción de venta de drogas como a reducir el creciente consumo. Asimismo, han proliferado los problemas de coordinación intergubernamental y la situación en general se caracteriza por la ausencia de una evaluación estratégica de los programas y sus impactos (Ramos, 2011: 75-78). Se puede afirmar, entonces, como lo hace Ramos (2011), que desde inicios de siglo «la política antidroga y de cooperación transfronteriza de Estados Unidos no ha sido eficaz para controlar la

${ }^{4}$ La iniciativa Mérida incluye recursos para Centroamérica, República Dominicana y Haití. A México se le asignaron 1.4 mil millones de dólares. 
oferta y demanda de drogas, lo que ha generado más inseguridad, violencia y posicionamiento del crimen organizado en las regiones fronterizas con México» (73).

Hoy en día, es posible identificar limitaciones serias de la Iniciativa Mérida y el fracaso de las políticas antidrogas bilaterales. Por ejemplo, en distintas regiones de la frontera México-Estados Unidos — sobre todo en las áreas colindantes con Chihuahua, Coahuila, Nuevo León y Tamaulipas - se observa claramente un incremento exponencial en los niveles de violencia relacionada con el crimen organizado, lo cual revela que las acciones de la Iniciativa Mérida han sido insuficientes. Por otro lado, se cuestiona si bajo este programa de cooperación «se han atendido los problemas de gestión que han mostrado deficiencias en cada país (incluyendo las naciones centroamericanas), ya que el mayor riesgo lo presentan los grupos delictivos que han desarrollado vínculos transfronterizos para sus actividades delictivas, financieras y de tráfico de armas». Por lo que respecta a México, quedan en evidencia las limitadas capacidades institucionales en los ámbitos local y estatal. En el plano intergubernamental, el gobierno federal mexicano no ha contado con una estrategia para fortalecer los esquemas de investigación, inteligencia y procuración de justicia. Asimismo, al parecer, «la cooperación transfronteriza en seguridad ha sido rebasada por la complejidad de la violencia regional» (Ramos, 2011: 84-85).

En lo que se refiere a la migración, el reforzamiento de la seguridad fronteriza en Estados Unidos ha tenido efectos contundentes que afectan sobre todo a quienes intentan cruzar a Estados Unidos sin documentos buscando mejores condiciones de vida. Por un lado, la migración ilegal a Estados Unidos parece haber disminuido finalmente después de varias décadas. ${ }^{5}$ Por otro

${ }^{5}$ Después de cuatro décadas, cuando el número de migrantes mexicanos en Estados Unidos llega a alrededor de 12 millones - siendo un gran número de estos ilegales-, la migración neta de México al vecino país del norte parece haberse detenido e incluso la tendencia ascendente parece haberse revertido recientemente de acuerdo a un análisis de datos gubernamentales de ambos países realizado por el Pew Hispanic Center. Este fenómeno parece ser el resultado de diversos factores que incluyen el debilitamiento de los mercados de trabajo y de la construcción de vivienda en particular en Estados Unidos derivado de la crisis económica; el reforzamiento de la seguridad fronteriza; el incremento masivo en las deportaciones en los

$158 \frac{\text { PRIMER SEMESTRE } 2014}{\text { MIGRACIÓN Y DESARROLLO NÚM. } 22}$ 
lado, «el desarrollo de redes criminales que realizan el tráfico de personas no ha disminuido sino que, por el contrario, aumenta». De acuerdo con Benítez (2011), esto se debe al hecho de que «al estar más protegida la frontera es mucho más difícil cruzarla de forma individual, por lo que los inmigrantes se ven obligados a contratar a las redes de traficantes para lograr su propósito. De igual manera, se ha consolidado la percepción de que las pandillas y las organizaciones criminales de México se han vuelto una amenaza a la seguridad nacional de Estados Unidos» (188).

En este contexto se observa un reforzamiento reciente de la securitización de la migración. Entre las consecuencias «no intencionadas» de este fenómeno se pueden mencionar (Rosemblum y Brick, 2011):

- Un incremento en los costos para llegar a Estados Unidos (la utilización de los servicios de un intermediario - el denominado coyote o pollero u otros grupos más organizados- por los migrantes indocumentados pasa de alrededor de 50\% en 1986 a 78\% en 1993).

- Un incremento en las cuotas para facilitar el traslado y el paso a Estados Unidos. Por ejemplo, éstas pasan de un promedio de 700 dólares por persona en 1983 a 2,800 dólares en los años 2007-2008.

- El traslado por áreas más inhóspitas y peligrosas, lo cual ha incrementado las tasas de mortalidad entre los migrantes de entre 10 muertes por año en la década de los ochenta a un promedio de entre 237 y 374 por año en el periodo 1995-2008.

- Un cambio en la demografía del migrante indocumentado. Antes de 1986, una gran parte de los migrantes eran hombres solteros y muchos eran migrantes temporales que regresaban a su lugar de origen. Ahora, más de 4 millones de mujeres y poco más de 1.1 millones de niños residen en Estados Unidos ilegalmente, y además un aproximado de 4 millones de niños nacen en Estados Unidos de padres cuya situación migratoria en el país es irregular.

- La aprobación o propuesta de legislaciones estatales antiinmigrantes del tipo de la SB-1070 de Arizona en diversos estados de Estados Unidos (como Alaba-

últimos años de la administración del presidente Obama; los mayores índices de peligrosidad asociados a los cruces fronterizos ilegales; y la caída en las tasas de natalidad en México (Passel, Cohn y González-Barrera, 2012). 
ma, Georgia, Indiana, Mississippi y Carolina del Sur). Este tipo de legislaciones han contribuido al «aumento de la imagen negativa del mexicano en la sociedad estadounidense, y [al] fortalecimiento de los temas de inmigración y seguridad fronteriza en el escenario político en ese país» (Armijo, 2011a: 9).

En resumen, la política de seguridad fronteriza después del 11 de septiembre, y ahora en la era de la denominada "guerra contra las drogas», ha resultado en una ligera disminución en el número de crímenes violentos y una reducción en el número de migrantes indocumentados en los últimos años en el caso de Estados Unidos. Pero en el caso de México se observa un incremento en la militarización y los niveles de violencia relacionados con el crimen organizado, principalmente en el noreste del país (Correa, 2013). Es importante destacar que no obstante los esfuerzos para reducir la migración ilegal hacia Estados Unidos en las últimas décadas, dichas políticas no han tenido el éxito esperado. Es posible pensar que la reciente disminución de los flujos migratorios hacia Estados Unidos se debe, en mayor medida, a condiciones externas — circunstancias económicas y creciente inseguridad en las zonas de tránsito-, y no únicamente al reforzamiento extremo en la seguridad fronteriza.

\section{Drogas, ViOLENCIA Y TRÁFICO DE PERSONAS en la frontera México-Centroamérica}

Diagnóstico de la frontera sur de México

Existen varios elementos que caracterizan la frontera México-Centroamérica, entre los que destacan su diversidad geográfica; ${ }^{6}$ la ausencia de procesos de industrialización y la dependencia de las actividades agrícolas, pecuarias y actividades diversas en las ramas de la construcción y los servicios; su porosidad; la debilidad del Estado en los países centroamericanos; la falta de

${ }^{6} \mathrm{Gran}$ parte de la frontera sur es fluvial y en la parte restante predominan las zonas montañosas y selvas principalmente.

$160 \frac{\text { PRIMER SEMESTRE } 2014}{\text { MIGRACIÓN Y DESARROLLO NÚM. } 22}$ 
profesionalización de los servicios de inteligencia y los cuerpos policíacos en estas naciones y México; el aumento inusitado del crimen organizado y del común en los últimos años; y la aparición de nuevas formas de delincuencia transnacional como las maras y otras pandillas (Armijo, 2011b; Benítez, 2011).

La principal problemática de la frontera sur de México reside en su porosidad. El tráfico de drogas, contrabando, tráfico de migrantes y tráfico de personas en distintas modalidades ponen de manifiesto las limitaciones relativas a la seguridad y la defensa de los derechos humanos en esta región del continente americano. Como explica Armijo (2011b), Centroamérica «se ha convertido en un puente para las actividades de los productores y traficantes de drogas después del relativo éxito de los operativos de Estados Unidos y Colombia para combatir las rutas aéreas y navales en el Caribe» durante la década de los noventa (45). Otro grave problema en esta región es el relacionado con la proliferación de pandillas como la Mara Salvatrucha 13 y Barrio 18.

Quizás, el fenómeno que actualmente requiere de mayor atención en esta región del hemisferio es el que tiene que ver con la migración irregular o indocumentada. La frontera México-Centroamérica «ha experimentado en los últimos 30 años un proceso de transformación marcado por la complejidad y el dinamismo de movimientos poblacionales de diverso tipo, inscritos en dinámicas locales y regionales en un área geográfica donde es posible visualizar crecientemente nuevos problemas de carácter interméstico, relacionados con el crimen organizado, la seguridad y los derechos humanos» (Armijo, 2011b: 35).

Dicha frontera es una zona de alta movilidad poblacional histórica. Debido a las guerrillas centroamericanas y las crisis económicas constantes, se observa un flujo importante de personas por esta región desde los años ochenta. En las últimas décadas se observa un marcado incremento de migrantes indocumentados en tránsito y la situación de seguridad en la región sufre un marcado deterioro.7 Como afirma Armijo (2011b), «el espacio uti-

${ }^{7}$ Respecto a la migración irregular, el Instituto Nacional de Migración (INM) ha señalado que cada año ingresan a México aproximadamente 140 mil indocumentados, la mayoría cen- 
lizado por las migraciones se ha tornado peligroso al verse invadido por actividades ilícitas, y las personas indocumentadas enfrentan riesgos inéditos durante su tránsito por territorio mexicano» (45). Asimismo, en "partes del territorio y en algunas vías de comunicación, las organizaciones criminales han sustituido al Estado o tienen comprados a miembros de las instancias de seguridad desplegadas para (supuestamente) evitar el tráfico de personas y drogas. Con ello han aparecido notables crisis humanitarias afectando la vida de los transmigrantes, principalmente centroamericanos, que se trasladan del sur al norte» (Benítez, 2011: 185).

La matanza de 72 migrantes en Tamaulipas en agosto de 2010 y la desaparición de entre 40 y 50 en Oaxaca en diciembre de ese mismo año son una clara muestra de que el problema se ha agravado. Los migrantes centroamericanos se han visto obligados «a transitar por nuevas rutas, más solitarias y peligrosas, así como a una mayor utilización de intermediaros o polleros, quienes a su vez han cambiado su perfil de "prestadores» de un servicio a traficantes que lucran con la necesidad de las personas. La cindustria» de la migración se ha fortalecido, y lo que para algunos es una estrategia de sobrevivencia, para otros es la posibilidad de un negocio ilícito (Armijo, 2011b: 46). Así, los migrantes centroamericanos se enfrentan a una situación de inseguridad y vulnerabilidad extremas. Armijo (2011b) describe esta situación de la siguiente manera:

Los abusos que sufren los migrantes por autoridades municipales, estatales y federales son principalmente el robo y la extorsión. El mayor número de agravios proviene de particulares y van desde el robo y el engaño, hasta las violaciones, el secuestro o la muerte. La presencia de mareros en la frontera sur se ha convertido en una pesadilla para los migrantes, sobre todo cuando algunos de ellos decidieron abandonar su país precisamente por estar amenazados por las maras. A las maras se han sumado pandillas compuestas principalmente por mexicanos, que aprovechan la situación de indefensión de los migrantes para asaltarlos y abusar de ellos. También se ha hecho mención de los «coyotes», gente

troamericanos, aunque funcionarios estatales y organismos civiles indican que la cifra podría ser mucho mayor, unos 400 mil por año (Martínez, 2011).

$162 \frac{\text { PRIMER SEMESTRE } 2014}{\text { MIGRACIÓN Y DESARROLLO NÚM. } 22}$ 
sin escrúpulos que además de engañar a los migrantes y no trasladarlos al destino acordado, participan en robos y violaciones. En este tráfico, las mujeres, niñas y niños son los más vulnerables (46).

Además, la presencia del crimen organizado en la frontera sur incrementa la vulnerabilidad de los migrantes con respecto a la trata de personas.

Los grupos considerados más vulnerables son mujeres, niños, niñas, indígenas y migrantes sin documentos. Predominan los casos relativos al comercio sexual, pero también se han reportado casos de niños, especialmente guatemaltecos, que trabajan en condiciones de explotación laboral en regiones agrícolas de Chiapas, en el servicio doméstico, en la mendicidad forzada, en el ambulantaje y en los basureros municipales (Armijo, 2011b: 47-48).

\section{Políticas actuales}

La situación general de (in)seguridad en la frontera México-Centroamérica se encuentra directamente relacionada con el incremento en los controles fronterizos en Estados Unidos y una mayor presencia de las fuerzas de seguridad en la frontera sur de México. En los últimos años se observa una mayor presencia militar en esta frontera. Dicho fenómeno se observa, quizás, desde la década de los noventa con la firma de Tratado de Libre Comercio de América del Norte (TLCAN), el movimiento zapatista y el inicio del endurecimiento de las políticas de inmigración de Estados Unidos (Armijo, 2011b: 42).

En este contexto se diseñan acciones selectivas de control y restricción de la inmigración, las cuales se reforzaron con los ataques terroristas a Estados Unidos el 11 de septiembre de 2001. «Con el objetivo de aumentar su capacidad de control de los flujos migratorios, México inició en julio de 2001 la implementación del Plan Sur, que contemplaba el fortalecimiento de acciones de intercepción de indocumentados desde el Istmo de Tehuantepec hasta la frontera sur» (Armijo, 2011b: 42-43). Se pueden mencionar también las políticas de «mano dura» aplicadas en Guatemala, Honduras y El Salva- 
dor para reducir los niveles de criminalidad y reducir el impacto de las pandillas. ${ }^{8}$ Por su parte, en fechas recientes se han observado deportaciones masivas de centroamericanos presos desde Estados Unidos.

No obstante lo anterior, en años recientes también se ha realizado esfuerzos importantes con el objeto de mejorar el marco institucional para la protección de los migrantes. Entre dichas mejoras, destacan el Plan Integral para la Frontera Sur, la creación de Grupos Beta para proteger la vida de los migrantes en situaciones de riesgo, la creación de la figura de Oficiales de Protección a la Infancia, la modernización de instalaciones migratorias, entre otras. Además, se han puesto en práctica programas de trabajadores agrícolas temporales, y una parte de la Iniciativa Mérida para mejorar la seguridad y combatir de manera más efectiva el crimen organizado se destinaría a países centroamericanos y del Caribe.

Debido al creciente problema del crimen organizado en México, el gobierno de este país ha reconocido la necesidad de brindar mayor atención a la frontera sur. En este contexto,

al inicio de sexenio del presidente Felipe Calderón en 2006 se propuso un Plan de Reordenamiento de la Frontera Sur, con el objetivo de articular los esfuerzos de distintas dependencias federales con los de las entidades federativas de la región y, así, incrementar el impacto de las acciones de los tres órdenes de gobierno. Mediante este plan se pretendía ordenar los flujos migratorios, mejorar la seguridad y combatir la impunidad (Armijo, 2011b: 48).

Otro importante esfuerzo del gobierno mexicano, que se supone tendrá un efecto importante sobre la seguridad de la frontera sur de México y en especial sobre la migración indocumentada que cruza por esta región e intenta llegar a Estados Unidos es la «nueva» Ley de Migración. ${ }^{9}$ Dicha legislación fue aprobada el 29 de abril de 2011 y crea un marco de garantías para prote-

${ }^{8}$ En particular hablamos de los programas «Mano Dura» y «Súper Mano Dura» de El Salvador, el «Plan Escoba» en Guatemala y «Libertad Azul» en Honduras.

${ }^{9}$ El texto completo de la nueva Ley de Migración se puede encontrar en la siguiente dirección: http://www.diputados.gob.mx/LeyesBiblio/pdf/LMigra.pdf.

$164 \frac{\text { PRIMER SEMESTRE } 2014}{\text { MIGRACIÓN Y DESARROLLO NÚM. } 22}$ 
ger los derechos de los migrantes en México. La Ley facilita y ordena los flujos migratorios desde y hacia México, privilegiando la protección y el respeto de los derechos humanos. Además, la nueva ley señala que en ningún caso una situación migratoria irregular representará la comisión de un delito, ni se prejuzgará la comisión de ilícitos. ${ }^{10}$ En este sentido, plantea un respeto irrestricto de los derechos de los mexicanos y extranjeros, «sea cual fuere su origen, nacionalidad, género, etnia, edad y situación migratoria».

\section{Evaluación de las políticas}

No obstante los esfuerzos recientes para proteger los derechos de los migrantes en México, la situación que éstos enfrentan continúa siendo muy delicada y la legislación insuficiente. En los últimos años, los flujos migratorios han disminuido, pero las condiciones de seguridad de los migrantes en las zonas fronterizas y en territorio mexicano en particular no han mejorado. La Comisión Interamericana de Derechos Humanos (CIDH), por ejemplo, advirtió que el principio de excepcionalidad de la detención ${ }^{11}$ de extranjeros indocumentados no está garantizado en la nueva Ley de Migración mexicana y tampoco en la práctica actual. La organización también señaló que existen «serias dificultades» para el ingreso de las organizaciones de la sociedad civil a las estaciones migratorias y que adicionalmente hay deficiencias en lo que respecta a la atención de grupos vulnerables (Martínez, 2011).

${ }^{10}$ La nueva legislación reconoce al Instituto Nacional de Migración (INM) como la instancia encargada de instrumentar y ejecutar la política migratoria. Y con el objeto de combatir la corrupción en el interior del instituto se da sustento al sistema de profesionalización y certificación de su personal, a través de un Centro de Evaluación y Control de Confianza. Además, esta legislación sustituye más de 30 calidades y características migratorias que actualmente considera la Ley General de Población, por sólo tres: visitante, residente temporal y residente permanente.

${ }^{11}$ Bajo este esquema, el argumento de que la persona representa una amenaza para la seguridad pública sólo es aceptable en "circunstancias excepcionales»; incluso la sola existencia de antecedentes penales no es suficiente para sustentar la detención de un inmigrante una vez que ha cumplido una condena (Martínez, 2011). 
En general, es posible afirmar que mientras que los gobiernos de México y Centroamérica continúan buscando soluciones para resolver la problemática en la frontera sur, las políticas recientes diseñadas para fortalecer la seguridad en esta región y evitar violaciones a derechos humanos han sido poco exitosas y difíciles de implementar. Por ejemplo, con el objeto de castigar el abuso en materia de derechos humanos en la frontera sur de México se crea en Chiapas, la primera Fiscalía Especializada en Atención a Delitos Cometidos en Contra de Inmigrantes. A su vez, en el lado guatemalteco se han enviado en fechas recientes un número creciente de representantes de la Procuraduría para la Defensa de los Derechos Humanos.

Sin embargo, dichas acciones no han tenido los resultados esperados. Incluso algunas organizaciones no gubernamentales (ONGs) han tenido que cerrar sus puertas como resultado de la presión que han sufrido por los grupos del crimen organizado interesados en el tráfico y explotación de migrantes. En este caso en particular, el problema parece radicar en que mientras que las oficinas ejecutivas de estas instituciones existen para documentar eventos y registrar quejas, no existen organizaciones diseñadas especialmente para ayudar a los migrantes que necesitan ayuda o se encuentran en situación de emergencia (Inkpen, 2011).

En general, es posible afirmar que la frontera México-Centroamérica ha pasado por «un largo periodo de abandono estatal que fue subsanado por una serie de políticas e intervenciones reactivas, focalizadas en resolver la emergencia, pero lejanas de una estrategia de gestión integral de las dinámicas fronterizas» (Armijo, 2011b: 45). En otras palabras, «la intermitente presencia gubernamental en la frontera sur no se ha traducido en políticas coherentes de largo alcance». En este contexto, de acuerdo con Armijo (2011a):

[L]os problemas de tráfico de drogas, violencia e inseguridad pública necesitan políticas de control, pero paralelamente se necesitan políticas migratorias eficientes que permitan el flujo ordenado de personas y faciliten la vida transfronteriza. El reto es el diseño y construcción de políticas fronterizas integrales, que alberguen estrategias diferenciadas para la atención de problemas en distintos ámbitos y para impulsar el desarrollo de la región (9).

$166 \frac{\text { PRIMER SEMESTRE } 2014}{\text { MIGRACIÓN Y DESARROLLO NÚM. } 22}$ 


\section{RECOMENDACIONES DE POLÍTICA Y COOPERACIÓN REGIONAL}

Las fronteras México-Estados Unidos y México-Centroamérica presentan diferencias fundamentales en relación con las políticas de seguridad fronteriza diseñadas e implementadas en la era reciente. La situación en la frontera sur de México es bastante delicada y es necesario el diseño de políticas efectivas para contrarrestar el tráfico de drogas, de armas y de personas, y, sobre todo, para proteger los derechos humanos de la población migrante que intenta cruzar a Estados Unidos de forma irregular y que se encuentra indefensa ante el crimen organizado.

En este caso, se buscan ejemplos y modelos exitosos para encontrar soluciones adecuadas. El caso de la frontera norte de México se utiliza frecuentemente como referencia. Sin embargo, los resultados de las políticas implementadas en esta región fronteriza no han sido siempre positivos, y los costos han sido, en su mayoría, para México. Además, las condiciones que viven ambas fronteras son muy distintas y, por lo tanto, sus necesidades de política difieren en gran medida. Finalmente, el papel de Estados Unidos cambia toda la dinámica fronteriza y es quien en realidad dicta muchas de las políticas para la región. La complejidad y limitaciones de adoptar en la frontera sur de México los modelos de seguridad fronteriza diseñados para la frontera norte se ilustran claramente a continuación:

México ha firmado numerosos acuerdos de cooperación con Guatemala y Belice con contenidos similares a los firmados con Estados Unidos, para tener un mayor control de las fronteras. Estos se sostienen en la creación de los Grupos de Seguridad Fronteriza Binacional. Las difíciles condiciones geográficas de la región — que es selvática y montañosa—, la deficiente cobertura de las instituciones y la corrupción constituyen escollos para que esos compromisos avancen en resguardar realmente la frontera. En el nivel retórico, los gobernantes se han comprometido a hacer legal la frontera sur de México, pero en la práctica este es un flanco vulnerable para la seguridad del país (Benítez, 2011: 182). 
Estados Unidos ha establecido una política de seguridad fronteriza relativamente exitosa, pero el nivel de cooperación con México no es una constante. En realidad, las políticas de seguridad fronteriza estadounidenses son más bien unilaterales. En términos generales, la cooperación en materia de seguridad y de combate al narcotráfico entre México y Estados Unidos en la frontera norte ha sido incapaz de reducir sustancialmente la inseguridad y la violencia en el lado mexicano. Entre los principales factores estructurales que han favorecido la violencia en la región están «la débil capacidad institucional de gobiernos locales y estatales, así como la ausencia de una cooperación eficaz entre México y Estados Unidos en materia de prevención y seguridad» (Armijo, 2011a: 9).

Y según explica Ramos (2011), «[1] os niveles de cooperación tanto intergubernamental del lado mexicano como transfronteriza con Estados Unidos han tenido serias limitaciones porque existen problemas de gestión (eficacia, efectividad, liderazgo, consensos, visión, estrategias, planeación y evaluación) que en su conjunto obstaculizan la implementación de políticas locales y una coordinación y planeación transfronteriza eficaz con los actores estadounidenses» (89). Sin la existencia de instituciones fuertes y efectivas para favorecer la seguridad en las fronteras, cualquier esfuerzo para resolver la problemática del narcotráfico, la trata de personas, la violencia fronteriza, el tráfico de migrantes y el lavado de dinero representará únicamente una solución fallida y costosa.

Es importante reconocer que no existe un solo modelo que deba aplicarse en todos los casos a todas las fronteras y, en especial, a estas dos fronteras tan diferentes. No es posible hacer una generalización sobre las políticas efectivas para resolver la problemática fronteriza actual sin analizar los factores causales que generan los distintos fenómenos en distintas regiones de la frontera y que afectan, de manera divergente, a los países involucrados en la compleja dinámica fronteriza actual, en la cual el crimen organizado ocupa, desafortunadamente, un papel primordial. Por ejemplo, aún cuando se reconoce que tanto los migrantes centroamericanos como los mexicanos tienden a presentar limitaciones y problemáticas similares en su trayecto hacia Estados Unidos, no es posible generalizar las medidas de control para ambas

$168 \frac{\text { PRIMER SEMESTRE } 2014}{\text { MIGRACIÓN Y DESARROLLO NÚM. } 22}$ 
fronteras, pues éstas presentan una serie de problemáticas y características distintas que hacen necesaria hacer una diferenciación de las políticas migratorias en las dos regiones del hemisferio.

Al mismo tiempo, es necesario plantear soluciones de más largo plazo que tengan que ver con el desarrollo regional, el crecimiento del empleo y la reducción de los altos niveles de pobreza y sobre todo de desigualdad que llevan a algunas personas a desplazarse de sus países a Estados Unidos en busca de mejores condiciones de vida. Las soluciones de corto plazo, algunas de ellas extremas, resultan medidas simplemente paliativas que no resuelven la problemática estructural. La evidencia reciente muestra que políticas de este tipo, tales como la construcción de un muro fronterizo a lo largo de la frontera México-Estados Unidos, o las políticas de mano dura para el abatimiento del crimen en algunos países centroamericanos, no han tenido el éxito ni la efectividad esperados, al tiempo que han representado un alto costo para los contribuyentes de las naciones donde han sido implementadas.

En este último sentido, por ejemplo, la política antidroga de Estados Unidos ha canalizado, desde hace aproximadamente dos décadas, enormes recursos financieros al control de las fronteras para evitar el cruce de marihuana y cocaína. Sin embargo, la realidad es que la droga sigue cruzando en los mismos niveles de hace diez o 15 años. Esto sigue sucediendo, según Ramos (2011), «porque los grupos delictivos tienen una gran capacidad estratégica, una capacidad para conciliar, corromper, generar alianzas y fomentar redes; actúan como verdaderas empresas con una visión estratégica. Es entonces un reto para la política pública generar precisamente esquemas mucho más flexibles y sobre todo más eficaces» (88).

En la actualidad observamos esfuerzos importantes en términos de cooperación para resolver la problemática fronteriza, tanto en la región que divide a México y Estados Unidos como en aquella que separa a México de Centroamérica. Sin embargo, dichos esfuerzos son limitados y se observan grandes problemas en las soluciones actuales diseñadas para resolver problemas comunes.

En la frontera norte, el principal problema reside en el diseño de políticas de seguridad fronteriza — sobre todo migratorias — de carácter unilateral, en 
particular por parte de Estados Unidos. La experiencia ha demostrado que las políticas unilaterales «orillan a la ilegalidad, aumentan los riesgos para la seguridad humana de los y las migrantes y favorecen el incremento de delitos asociados a la migración» (Armijo, 2011a: 8). En lo que respecta a la frontera sur, como señala Benítez (2011), «tanto en México como en América Central las capacidades de los gobiernos son muy reducidas, sobre todo ante el poder acumulado por las agrupaciones criminales. A ello se agrega una debilidad endémica de los sistemas políticos en países como Guatemala, Honduras y El Salvador, que impide el desarrollo de capacidades para neutralizar a los traficantes de personas y drogas» (192).

Tomando en cuenta lo anterior, se hacen necesarios los programas de cooperación regional en defensa, inteligencia, justicia y seguridad pública entre México, Centroamérica y Estados Unidos. En realidad, «solamente la cooperación multilateral en materia de seguridad y migración puede generar respuestas que aborden la complejidad de estos procesos» (Armijo, 2011a: 8). Al mismo tiempo, se debe garantizar la prevención, detección, investigación, sanción y reparación efectiva de abusos a migrantes. Además, deben implementarse mejoras en la comunicación y tecnologías de información; reforma policial y del sistema penitenciario; así como mejoras en los sistemas de inteligencia.

Un enfoque integral como este debería de preocuparse, además, por los aspectos sociales, la prevención y la reducción del consumo de drogas en Estados Unidos; esto implicaría avanzar en el tema de la corresponsabilidad y reducir los niveles de desconfianza. La gestión y la cooperación entre los tres niveles de gobierno, tanto de México como de Estados Unidos, es también fundamental para definir «una estrategia común y eficaz, que genere impactos en la reducción sustancial del narcotráfico, la violencia y la inseguridad» (Ramos, 2011: 90).

Los retos para solucionar la problemática actual en las dos fronteras de México son enormes e involucran a una gran cantidad de actores. Las oportunidades de una mayor y mejor colaboración son también evidentes, sobre todo ahora que algunos países de la región —en particular México y Estados Unidos - inician una nueva fase en su configuración política derivada de

$170 \frac{\text { PRIMER SEMESTRE } 2014}{\text { MIGRACIÓN Y DESARROLLO NÚM. } 22}$ 
procesos electorales recientes. El futuro de la Iniciativa Mérida —o del programa que la sustituya - depende de la voluntad de los gobiernos de la región, recordando que los principales problemas en materia de seguridad fronteriza (en las dos fronteras que aquí se analizan) sólo podrán abordarse a través de la cooperación de los países involucrados.

\section{RefERENCIAS}

Armijo, Natalia (2011a), «Introducción», en Natalia Armijo (ed.), Migración y seguridad: nuevo desafío en México, México, CADESE. (2011b), «Frontera sur de México: los retos múltiples de la diversidad», en Natalia Armijo (ed.), Migración y seguridad: nuevo desafí en México, México, CADESE.

Benítez, Raúl (2011), «México, Centroamérica y Estados Unidos: migración y seguridad», en Natalia Armijo (ed.), Migración y seguridad: nuevo desafío en México, México, CADESE.

CorreA, Guadalupe (2013), «Security, migration, and the economy in the Texas-Tamaulipas border region: the "real" effects of Mexico's drug war», Politics \& Policy, vol. 41, núm. 1.

InKen, Christopher (2011), "Security and human rights issues on the Guatemalan/ Mexican border", Center for strategic and international studies (CSIS), Washington, DC, 5 de enero, en http://csis.org/blog/security-and-human-rights-issues-guatemalanmexican-border-chris-inkpen (consultado el 15 de septiembre 2012).

Martínez, Fabiola (7 de agosto de 2011), "La nueva ley migratoria no garantiza la excepcionalidad de indocumentados: CIDH», La Jornada, en http://www.jornada.unam.mx/2011/08/07/politica/006n1 pol (consultado el 19 de septiembre 2012).

Passel, Jeffrey, D’Vera Cohn y Ana González-Barrera (2012), Net migration from Mexico falls to zero-and perhaps less, Washington, Pew Hispanic Center.

Ramos, José María (2011), "Gestión de la seguridad en la frontera norte e Iniciativa Mérida: antecedentes y desafíos», en Natalia Armijo (ed.), Migración y seguridad: nuevo desafío en México, México, CADESE.

Rosemblum, Marc R. y Kate Brick (2011), U.S. immigration policy and Mexican/Central American migration Flows: then and now, Washington, Migration Polity Institute. 
